\title{
ADEQUAÇÃO DO FLUXO DAS ROUPAS SUJAS PARA DESINFECÇÃO ${ }^{1}$
}

\author{
ADEQUACY OF THE FLOW OF DIRTY CLOTHES FOR DISINFECTION
}

\author{
ADECUACIÓN DEL FLUJO DE LA ROPA SUCIA PARA LA DESINFECCIÓN.
}

\author{
Mônica Monninger Fernandes de Góes Vaz de Lima \\ Universidade de Guarulhos \\ monicamonninger@hotmail.com
}

Antônio Carlos Estender

Pontifícia Universidade Católica de São Paulo

estender@uol.com.br

\author{
Andreia de Torres \\ Universidade de Guarulhos \\ torres.andreia@uol.com.br
}

\begin{abstract}
RESUMO
No processo de desinfecção do enxoval do centro cirúrgico realizado no Hovet há as seguintes desconformidades: roupas contaminadas são armazenadas de maneira inadequada. $\mathrm{O}$ transporte das mesmas também é feito de maneira incorreta. Este projeto se propõe a traçar novos fluxos da lavanderia para que ela cumpra seu principal objetivo: transformar roupa suja e contaminada em roupa limpa, em tempo adequado, na quantidade necessária, com segurança e qualidade. A lavagem também deve ser realizada de forma a garantir e assegurar a eliminação de substancias irritantes, incluindo todos os produtos necessários para limpeza e remoção das impurezas existentes na roupa com ações voltadas à prevenção de riscos e à saúde dos trabalhadores. Foi realizada uma revisão de literatura usando-se os seguintes passos: identificação, amostragem ou busca na literatura, extração dos estudos incluídos e sua avaliação e interpretação dos resultados. Na retirada da roupa suja, deve-se proceder com o mínimo de agitação e manuseio, observando-se as precauções padrão, independente da sua origem. Isso ajuda a prevenir acidentes e dispersão de microorganismos para o ambiente e pacientes. Neste sentido está indicada apenas a pesagem e nunca a contagem de roupas sujas. É desejável que o transporte da roupa limpa e suja seja efetuado em carros separados. Porém, se o serviço dispõe apenas de um carro para esse fim, pode-se optar pela sua lavagem e desinfecção após o transporte da roupa suja e antes do transporte da roupa limpa, no caso desta estar embalada. O serviço deve possuir por escrito todo o processo de limpeza e desinfecção desses carros.
\end{abstract}

Palavras-Chaves: Adequação do fluxo da roupas sujas para desinfecção

\begin{abstract}
In the process of disinfection of the trousseau from the Surgical Center at Hovet, there is the following nonconformities: contaminated clothing are stored improperly. The transport of the same is also done incorrectly. This project proposes to draw new flows of laundry so she fulfills the main goal: to transform dirty and contaminated clothes clothing in clean clothes, in timely manner and the necessary amount, in a safety process with quality. The washing should also be performed to ensure and assure the elimination of irritating substances, including all products needed for cleaning and removal of debris from the machine; with actions focused on risk prevention and the health from the workers. We performed a literature review using the following steps: identification, sampling or literature search, extraction of included studies and their evaluation and interpretation of results. In the time to remove the dirty clothes, you should proceed with minimal agitation and handling, observing standard precautions, regardless of their origin. This helps prevent accidents and dispersion of microorganisms into the environment and the patients. In this case, is showing just the weighing and never the counting for the dirty clothes. It is desirable that the transport of clean and dirty clothing is made
\end{abstract}

\footnotetext{
${ }^{1}$ Submetido em 21 de dezembro de 2012. Aceito em 13 março de 2013. O artigo foi avaliado segundo o processo de duplo anonimato além de ser avaliado pelo editor. Editores responsáveis: Márcio Augusto Gonçalves e Lucas Maia dos Santos. Reprodução parcial ou total e trabalhos derivativos permitidos com a citação apropriada da fonte.
} 
in separate cars. However, if the service has only one drive for this purpose, the vehicle must be wash and disinfected after transporting the laundry and before to transport the clean clothes, in this case the clothes must be packaged. The service must have written the entire process of cleaning and disinfecting the cars.

Key-words: Adequacy of the flow of dirty clothes for disinfection.

\section{RESUMEN}

En el proceso de desinfección del centro quirúrgico ajuar Hovet se llevó a cabo en los siguientes incumplimientos: la ropa contaminada se almacenan inadecuadamente. El transporte de la misma también se realiza incorrectamente Este proyecto se propone trazar nuevos flujos de ropa que cumple su principal objetivo: transformar ropa contaminada y póngase ropa limpia, en el momento adecuado, en la cantidad requerida, con seguridad y calidad. El lavado también se debe realizar con el fin de garantizar y asegurar la eliminación de sustancias irritantes, como todos los productos necesarios para la limpieza y la eliminación de residuos de la máquina con las acciones encaminadas a la prevención de riesgos y de los trabajadores de salud Se realizó una revisión de la literatura mediante los siguientes pasos: identificación, muestreo o búsqueda en la literatura, la extracción de los estudios incluidos y su evaluación e interpretación de los resultados. Sobre la retirada de la ropa sucia, se debe proceder con la agitación y la mínima manipulación, observando las precauciones estándar, independientemente de su origen. Esto ayuda a prevenir los accidentes y la dispersión de los microorganismos en el medio ambiente y los pacientes En este sentido, sólo se indica para el pesaje y contando ropa nunca sucios Es deseable que el transporte de la ropa sucia y limpia se hace en coches separados Sin embargo, si el servicio sólo tiene un coche para este fin, se puede optar por su lavado y desinfección después de transportar la ropa y antes del transporte de ropa limpia, en este caso está lleno. El servicio debe haber escrito todo el proceso de limpieza y desinfección de estos vehículos.

Key Words: Adecuación del flujo de la ropa sucia para la desinfección

\section{Introdução}

O método de purificar lençóis e cobertores de pacientes através de fumigações e vapor de água já era recomendado por Characa, médico indiano, entre os atributos de um bom hospital, desde o século três antes de Cristo. Florence Nightingale, do Barrack Hospital, após verificar que as roupas entregues aos civis para serem lavadas, além de demorarem muito, voltavam mais sujas, sendo muitas vezes necessário destruí-las a fogo para que não trouxessem mais malefícios aos doentes, resolveu dar fim a esses contratos e com ajuda dos engenheiros militares organizou um bom serviço de lavanderia hospitalar, instalando uma caldeira.

Foi detectado por Semmelweis que a reutilização de roupas sujas por diferentes doentes causava novos surtos e isso o levou a recomendar que as roupas fossem lavadas e cloradas. Essas experiências, devido a sua coerência, tiveram grande papel no desenvolvimento das lavanderias hospitalares e são mantidos até hoje.

Dois princípios básicos norteiam os controle de infecções em uma lavanderia hospitalar: não agitar e remover ou destruir os microrganismos contaminantes. Isso porque o ser humano elimina em torno de 3.000 a 60.000 bactérias por minuto que se aderem ao tecido. Tendo-se relatos de contagens que vão de $10^{6}$ a $10^{8}$ bactérias por $\mathrm{cm}^{2}$ de tecido. Sendo que o contatos direto com estas roupas contaminam equipamentos, mãos e o uniforme dos profissionais de saúde. Pasteur provou que as infecções são causadas por micróbios, com isso Lister aprofundou a área bacteriana e revolucionou a forma de preparo dos instrumentais e produtos utilizados nas cirurgias e curativos. Isso levou o Serviço de Lavanderia a ter mais cuidado com os critérios de prevenção das infecções causando o surgimento de dois serviços específicos: Esterilização de Materiais e Lavanderia Hospitalar.

Embora os hospitais busquem novas tecnologias e profissionais mais especializados para as funções administrativas e técnicas a falta de conhecimentos específicos de lavanderia, no que diz respeito às especificações de arquitetura e engenharia, leva os construtores instaladores a cometerem erros, como inadequada localização de equipamentos e instaladores não condizentes com o serviço. 
Nosso objetivo é estabelecer um fluxo de entrada e saída de roupa suja e limpa, adequado segundo a legislação vigente pela Anvisa e Ministério da Saúde. Como recolher a roupa, a maneira exata de acondicionar este material contaminado em hampers e como tirar este material para que tenha o mínimo de contaminação.

Este Projeto visa orientar e instruir as condições estabelecidas para a melhoria dos serviços em lavanderia hospitalar envolvendo o processamento de roupas e tecidos em geral em todas as suas etapas, sua utilização, transporte e o seu retorno em condições ideais de reuso, sob situações higiênico-sanitárias adequadas, para atender o Hovet de acordo com as normas que regulamentam a prestação desses serviços. Implantação correta, com reforma adequada e uma administração dentro da técnica atualizada.

Implantação correta, com reforma adequada e uma administração dentro da técnica atualizada. Limpeza ambiente com menor carga de contaminação possível, contribuindo com a redução da possibilidade de transmissão de infecções oriundas de fontes inanimadas. As infecções relacionadas à assistência à saúde representam um risco substancial à segurança do paciente em serviços de saúde. Há evidências mostrando que vários patógenos como Staphylo-coccus aureus resistente à meticilina, Enterococos resistente à vancomicina e outros contaminam superfícies e equipamentos (bombas de infusão, e estetoscópio e outros) mais freqüentemente manuseados pelos profissionais e pacientes. Sendo assim, falhas nos processos de limpeza e desinfecção de superfícies podem ter como conseqüência a disseminação e transferência de microrganismos nos ambientes dos serviços de saúde, colocando em risco a segurança dos pacientes e dos profissionais que atuam nesses serviços. A limpeza e desinfecção de superfícies, objetos e roupas em serviços de saúde são elementos primários e eficazes nas medidas de controle para romper a cadeia epidemiológica das infecções e diminuir riscos.

A definição de risco engloba uma variedade de medidas de probabilidades incluindo aquelas baseadas em dados estatísticos ou em julgamentos subjetivos. De acordo com Costa, risco é a probabilidade de ocorrência de um evento danoso à saúde, relacionado com objetos concretos sob controle sanitário.

Ainda como descrito por Costa, "os riscos à saúde se instalam em qualquer momento do processo produção-consumo". Também refere que a produção - especialmente em determinados ramos de atividade - gera riscos à saúde do trabalhador e ao meio ambiente, e que nesse percurso, intencionalmente ou por falhas no processo, podem ser adicionados riscos aos objetos de consumo. Refere que isso é agravante para as questões de saúde, pois, no caso de produtos de interesse sanitário, muitos deles, por si mesmos, já contêm certo quantum de riscos. A mesma autora afirma que "na prestação de serviços, direta ou indiretamente relacionados com a saúde, também são gerados riscos, potencialmente multiplicados pelo uso concomitante de várias tecnologias em ambiente exposto a diversos fatores de riscos".

Este trabalho visa colher e acatar todas as ações capazes para eliminar, riscos à saúde segundo conceitos de biossegurança hospitalar e de intervir nos problemas sanitários decorrentes do meio ambiente, da produção e circulação de bens e da prestação de serviços de interesse da saúde.

Metodologia: Foi realizada uma revisão de literatura. Para a elaboração desta revisão, foram percorridos os seguintes passos: identificação do tema, amostragem ou busca na literatura, extração dos estudos incluídos e sua avaliação, interpretação dos resultados e síntese do conhecimento obtido. Na seleção dos materiais incluídos na revisão, utilizou-se a internet para acessar as bases de dados Simpoi, Semead, Scientific Eletronic Library Online (SciELO), entre outros. Os critérios de inclusão foram: materiais que reportassem, direta ou indiretamente, a temática, publicados entre 2000 a 2012, nos idiomas inglês, português e 
espanhol, exclusivamente desenvolvidos no Brasil. A busca dos dados e a análise dos resultados foram feitas entre período de agosto e novembro de 2012.

O estudo esta estruturado em cinco seções além desta introdução. Na primeira seção é discutida a questão do referencial teórico; adequação do fluxo seguro e gerenciamento de risco. A seguir, é detalhado os aspectos metodológicos; estudo de caso e pesquisa bibliográfica. Na terceira seção, foi apresentado a Hovet que se trata de um Hospital escola, que conta com serviços na área de clinica médica, clinica cirúrgica, na quarta seção são apresentados a analise de dados com enfoque em utilizando dos conceitos de biossegurança hospitalar. Na ultima secção são expostos as conclusões finais que foram garantir atendimento com qualidade aos pacientes e a todos os colaboradores do Hovet

\section{Referencial Teórico}

\subsection{Adequação do fluxo Seguro}

Segundo Costa, E.A. deve haver uma orientação escrita e prática, referente às atividades envolvidas no processamento de roupas de serviços de saúde, tendo como foco os riscos associados a essas atividades, uma vez que as ações desse sistema baseiam-se no controle de riscos definido pela Lei n. 8.080 de 19 de setembro de 1990 Entende-se por vigilância sanitária um conjunto de ações capazes de eliminar, diminuir ou prevenir riscos à saúde e de intervir nos problemas sanitários decorrentes do meio ambiente, da produção e circulação de bens e da prestação de serviços de interesse da saúde, abrangendo:

I - o controle de bens de consumo que, direta ou indiretamente, se relacionem com a saúde, compreendidas todas as etapas e processos, da produção ao consumo;

II - o controle da prestação de serviços que se relacionam direta ou indiretamente com a saúde (Diário Oficial da União, Brasília, 20 set. 1990).

Segundo Murahovschi et al., os controles de risco e de qualidade são inseparáveis e a diminuição da qualidade da assistência prestada leva ao aumento do risco para usuários e trabalhadores. Na vigilância sanitária de serviços de saúde, aos conceitos mais amplos de gerenciamento de risco e qualidade, agrega-se ao marco teórico uma abordagem já tradicional da administração em saúde: os conceitos de estrutura, processo e resultados de Donabbedian, absorvidos da teoria de sistemas.

Ainda como descrito por Costa, os riscos à saúde se instalam em qualquer momento do processo produção-consumo. Cabe salientar que a produção-especialmente em determinados ramos de atividade gera riscos à saúde do trabalhador e ao meio ambiente, e que nesse percurso, intencionalmente ou por falhas no processo, podem ser adicionados riscos aos objetos de consumo. Refere que isso é agravante para as questões de saúde, pois, no caso de produtos de interesse sanitário, muitos deles, por si mesmos, já contêm certo quantum de riscos. A mesma autora afirma que "na prestação de serviços, direta ou indiretamente relacionados com a saúde, também são gerados riscos, potencialmente multiplicados pelo uso concomitante de várias tecnologias em ambiente exposto a diversos fatores de riscos". 


\subsection{Gerenciamento de Risco}

De acordo com Costa a definição de risco engloba uma variedade de medidas de probabilidades incluindo aquelas baseadas em dados estatísticos ou em julgamentos subjetivos. Risco é a probabilidade de ocorrência de um evento danoso à saúde, relacionado com objetos concretos sob controle sanitário.

Segundo Murahovschi et al. avançam no conceito definindo risco como a probabilidade de ocorrência de um evento adverso que, no caso dos serviços de saúde, afeta a integridade do paciente, da equipe de saúde ou da comunidade em que o serviço está inserido. $\mathrm{O}$ risco é determinado por dois componentes: $\mathrm{O}$ risco inerente é aquele que advém do próprio processo ou procedimento em questão, seja por limitações tecnológicas ou do "estado da arte" dessa atividade, ou por características próprias do paciente que está sendo submetido a um processo ou procedimento. O risco adquirido é o risco adicionado, ou seja, uma parcela que não é decorrente da natureza do processo, procedimento ou daquele que recebe esta ação. $\mathrm{O}$ risco adquirido pode resultar da falta de qualidade na assistência à saúde. Por isso, a vigilância sanitária tem trabalhado no sentido de reduzir o risco atribuído às diferentes condições de estrutura e de processo de trabalho; Observa-se, portanto, que o conceito de risco é o principal referencial teórico das ações de vigilância sanitária e que a eliminação de riscos refere-se a uma minimização de sua manifestação a níveis estatisticamente não significantes.

A avaliação e o gerenciamento de risco são os elementos fundamentais para as ações da vigilância sanitária. Segundo Luchese, a "Avaliação de Risco, de natureza mais científica (estatística e epidemiológica), consiste no uso de bases concretas de dados para definir os efeitos de uma exposição (indivíduos ou população) a materiais ou situações; ou seja, conhecer a relação causa-efeito e possíveis danos ocasionados por um determinado agente e o Gerenciamento de Risco, ação de orientação político-administrativa, é o processo de ponderar as alternativas de políticas e selecionar a ação regulatória mais apropriada, integrando os resultados da avaliação de risco com as preocupações sociais, econômicas e políticas para chegar a uma decisão; decide o que fazer com risco avaliado e se ele pode ser aceitável".

Pode-se identificar experiências bem sucedidas na prevenção e controle de riscos em serviços de saúde, em geral acumulando ações de educação, regulamentação, incentivo a sistemas externos de qualidade, estruturação de redes sentinelas, sistemas de informação, notificação e investigação de eventos adversos. Para Murahovschi et al., os controles de risco e de qualidade são inseparáveis e a diminuição da qualidade da assistência prestada leva ao aumento do risco para usuários e trabalhadores. Na vigilância sanitária de serviços de saúde, aos conceitos mais amplos de gerenciamento de risco e qualidade, agrega-se ao marco teórico uma abordagem já tradicional da administração em saúde: os conceitos de estrutura, processo e resultados de Donabbedian, absorvidos da teoria de sistemas.

A unidade de processamento de roupas realiza diversas atividades que envolvem riscos à saúde do trabalhador, do usuário e do meio ambiente e, por isso, é alvo da ação de regulação da vigilância sanitária. De acordo com Prochet, o serviço de processamento de roupas é uma área da saúde pouco conhecida e estudada, que pode, entretanto, representar um grave problema, principalmente pelas condições e riscos que oferece ao trabalhador desse setor, o qual está sujeito aos riscos físicos, químicos, biológicos, ergonômicos, psicossociais e de acidentes. Dentro dessa classificação destacam-se aqueles relacionados ao uso de produtos químicos, à manipulação e à operação dos equipamentos, à inadequação da infraestrutura física da unidade e à organização do trabalho. Diante do exposto, conclui-se que a qualidade das atividades da unidade de processamento de roupas em serviços de saúde está intrinsecamente relacionada ao gerenciamento dos riscos associados, o que requer, cada vez mais, conhecimento e divulgação científica sobre a temática. 


\section{Aspectos Metodológicos}

A metodologia a ser implantada será primeiramente criação de uma planilha para ser usada no setor de esterilização, para mensurar a quantidade de roupa suja que será gerada por dia. Com estes dados implantar uma rotina de fluxo desde a saída da roupa suja da sala cirúrgica, acondicionamento adequado, retirada, transporte até a lavanderia, processamento da roupa e entrega da mesma no hospital. O processo de melhorias será implantado por etapas descritas abaixo:

- Criação de uma planilha de peso de roupa suja ( saber quanto o hospital gera de roupa suja por dia);

- Implantação de uma rotina de fluxo desde a saída da roupa suja da sala cirúrgica, acondicionamento adequado, retirada, transporte até a lavanderia, processamento da roupa e entrega da mesma no hospital;

- Criação de Protocolo passa a passo de como será todo o processo.

O Método Estudo de Caso pode ser utilizado em estudos empíricos que investigam fenômenos atuais e permite realizar uma investigação em profundidade de uma realidade, preservando as características significativas dos eventos da vida real. Este método permite a união de conhecimentos advindos de teorias com experiências práticas e pesquisas de campo. É adequado para investigar fenômenos sociais e baseia-se em várias fontes de evidência para estudar e explicar fenômenos sociais complexos, dentro de seu contexto real (YIN, 2001).

2 O estudo de casos é um método de pesquisa que tem várias aplicações, dentre as quais se destacam procurar explicar as variáveis causais de determinado fenômeno por meio de uma intervenção em uma situação da realidade que é muito complexa para ser identificada por meio de um levantamento ou experimento, descrever a situação real do contexto no qual está sendo feita a intervenção e pode, também, ser usado para estudar e explorar situações da vida real cujos limites não estão claramente definidos (PATTON, 1980).

3 Para Schram (1971), o que justifica a escolha deste método é a sua própria essência, a tentativa de esclarecer as estratégias implementadas, atentando porque elas foram adotadas, como foram implantadas e desenvolvidas e quais os resultados, pois esta estrutura de estudo tem com objetivo identificar e interpretar um fenômeno; além disso, o problema da pesquisa em questão está relacionado com pessoas, em uma perspectiva comportamental e sua investigação não atingiria o grau de profundidade necessário, se fosse realizada com algum método baseado simplesmente em pesquisas quantitativas.

O método científico para a elaboração deste artigo seguiu os passos da revisão de literatura e incluiu: identificação do tema, levantamento bibliográfico, seleção de textos, estruturação preliminar e estruturação lógica do estudo. A revisão de literatura proporciona aos leitores os antecedentes para a compreensão do conhecimento atual sobre um tópico e esclarece a importância de novos estudos. A revisão de literatura serve assim, como função integradora e facilita o acúmulo de conhecimento. 
A busca de materiais sobre o tema foi realizada no período de Julho a Dezembro de 2012 em bases de dados LILACS e Scielo. Foram utilizados como critérios de seleção materiais (artigos e livros-texto) publicados que abordassem o tema.

Este método de estudo tornou-se mais convincente quando apresentado na forma de casos múltiplos em detrimento do estudo de caso único (GODOY, 2005).

\section{O Hospital Veterinário da Universidade de Guarulhos (HOVET)}

O HOVET Hospital Veterinário da Universidade de Guarulhos foi fundado em 2000. É um dos principais hospitais da região, se tornando referencia no município de Guarulhos. Por ser um hospital escola, conta com serviços na área de clinica médica, clinica cirúrgica, setor de imagem, laboratório de analises clinica, em pequenos animais, para animais de grande porte, conta com serviço de internação.

Além de servir com qualidade e garantia o seu público, o hospital tem como função servir de campo de estágio para o estudante busca conhecimento antes de sua formação, ou seja, é neste que o acadêmico de Medicina Veterinária pode desempenhar atividades na prática que são passadas, em aula, contando sempre com ajuda de Professores, Mestres e Doutores.

De uma forma geral, todos os setores contam com profissionais capacitados, prontos a atender qualquer emergência; com enfoque no setor de Laboratório de Análises Clínicas, este conta com equipamentos e mão de obra capacitada para atender exclusivamente a demanda de exames requeridos no hospital, não aceitando pedidos de realização de exames externos.

Cabe salientar que os serviços do HOVET assim como as consultas e cirurgias não são gratuitos, mas atende a comunidade com preços bem inferiores aos cobrados em clinicas particulares, valor que se destina unicamente a compra de materiais, proporcionando ao Hospital auto sustentabilidade sem fins lucrativos.

\section{Analise de Dados}

Implantação correta da rouparia, com reforma adequada da mesma e uma administração dentro da técnica usada atualmente, utilizando os conceitos de biossegurança hospitalar humano. Aproveitando a localização privilegiada, o hospital esta a beira de uma importante rodovia no estado de São Paulo, Rodovia Presidente Dutra, com fácil acesso e próximo a outras rodovias importantes como a Rodovia Fernão Dias, Carvalho Pinto, etc. Nossa infraestrutura é dotada de profissionais qualificados, há a presença de uma enfermeira qualificada no setor de esterilização de materiais.

Para que o setor de esterilização funcione adequadamente há a necessidade de treinamento periódico. O fato da Universidade de Guarulhos possuir um Hospital Veterinário favorece a aplicação de novas técnicas e treinamento continuo, o que é importante para o desenvolvimento e aperfeiçoamento profissional não só dos alunos e profissionais do 
HOVET, como para outros estudantes e profissionais de estabelecimentos de ensino que procurem o campus participarem e conhecerem as técnicas de biossegurança.

Apesar do Médico Veterinário saber que é importante a esterilização tanto de instrumentais, como de roupas, compressas, campos cirúrgicos, propés, aventais, gorros, para um bom serviço de atendimento cirúrgico, não se preocupam em se manter informados, de novas técnicas, de esterilização. A Anvisa e o Ministério da Saúde estão constantemente estudando novas tecnologias para minimizar o risco a saúde, embora se utilizem biotecnologias de ambientes hospitalares humanos em hospitais veterinários, por não ter ainda uma regulamentação especifica para o Médico Veterinário. A prevenção da ocorrência de infecção hospitalar depende dos profissionais da saúde e de programas eficientes de controle de infecções que norteiem as ações desses profissionais, bem como, de medidas de identificação e redução dos patógenos no ambiente hospitalar e de vigilância de doenças entre clientes e a equipe de saúde. Tais programas requerem educação e cooperação de toda equipe envolvida no cuidado ao cliente, seja em ambiente hospitalar ou ambulatorial.

\section{Considerações Finais}

Apesar da roupa suja possuir um grande número de micro organismos patogênicos, o risco de transmissão de doenças é praticamente inexistente se ela for corretamente manipulada, processada e não possui papel relevante na cadeia epidemiológica das infecções hospitalares. Alguns estudos na literatura apontaram a roupa hospitalar como provável fonte de infecção, mas em todos os casos relatados as principais medidas de controle foram negligenciadas.

A combinação de fatores mecânicos, térmicos e químicos resulta na ação antimicrobiana do processo de lavagem. A diluição e a agitação da roupa removem substancial quantidade de micro organismos. A ação dos detergentes promove a suspensão e a remoção de sujidades e também possui propriedade antimicrobiana. A temperatura elevada da água e/ou o uso de alvejantes também contribui para a destruição microbiana. Além disso, a mudança do $\mathrm{pH}$ de 12 para 5, na etapa de neutralização, e as etapas de secagem e calandragem também promovem uma ação antimicrobiana adicional. Para que o serviço seja implantado de forma adequada, segue abaixo fluxo de como se pretende trabalhar com o enxoval da HOVET.

\section{Coleta da roupa suja no setor de expurgo do HOVET:}

Para a efetiva execução dos serviços de recebimento de roupas hospitalares, o HOVET deverá disponibilizar na unidade hospitalar:

> Uma (1) balança Digital com laudo de aferição válido por 6 meses emitido por empresa especializada do ramo.

$>$ Contêiner com tampa lavável.

$>$ Sacos hampers de tecido ou de plástico descartáveis.

- A coleta será feita no setor de expurgo do HOVET, por funcionário devidamente treinado, uniformizado, e equipado com os EPI's (Equipamentos de Proteção Individual).

- A coleta será feita com a utilização de carrinho tipo "contêiner" com tampa, lavável, com dreno para eliminação de líquidos e devidamente identificado. 
- As roupas retiradas, diariamente, deverão ser devidamente acondicionadas, conforme normas de biossegurança.

- A periodicidade de retirada da roupa deverá ser de duas vezes ao dia, em horário estabelecido pelo HOVET.

- O deslocamento da roupa suja até o veículo que a transportará até as dependências da lavanderia deverá ser feito, por meio da "rota de roupa suja", observando-se que, em hipótese alguma haja cruzamento entre roupa limpa e roupa suja.

\section{Separação e retirada da roupa suja:}

- A roupa suja deverá ser separada seguindo critérios e técnicas estabelecidas de acordo com o tipo de tecido e tipo de sujidade;

- O funcionário que faz a separação da roupa deve usar máscara, proteção ocular, avental, botas e luvas de borracha cobrindo os braços;

- Para diminuir a contaminação dos profissionais e do ar, a roupa deve ser manuseada com um mínimo de agitação possível. Além disto, para evitar acidentes com objetos perfuro cortantes inadvertidamente coletados, é recomendável puxar as roupas pelas pontas, cuidadosamente, sem apertar nem recolher várias peças de uma vez.

- Deverá ser elaborado um relatório diário pela lavanderia, informando o peso da roupa retirada (em KG). Este relatório deverá ser aprovado pelo funcionário do HOVET.

\section{Transporte da roupa para as dependências da lavanderia:}

- O transporte da roupa suja do HOVET até as dependências da lavanderia deverá ser feito por veículo adequado devidamente adaptado à natureza da carga.

- A lavanderia tem obrigação de manter o veículo em bom estado e realizar a manutenção preventiva e corretiva que se julgue necessária para o bom funcionamento do mesmo e prevenção de potenciais acidentes.

\section{Recebimento e acondicionamento roupa suja na lavanderia:}

- O recebimento e acondicionamento da roupa suja na lavanderia devem obedecer aos procedimentos constantes no Manual de Processamento de Roupas de Serviço de Saúde: Prevenção e Controle de Risco da Agência Nacional de Vigilância Sanitária - 2007, que atualiza o Manual de Lavanderia Hospitalar do Ministério da Saúde - 1986 e suas atualizações.

Aplicando-se adequadamente uma rotina para retirada da roupa suja e retorno da mesma, acredita-se que além de melhorar as condições de uso, haverá uma maior segurança para os profissionais envolvidos no processo.

Este trabalho visa a melhoria das condições atuais no Hovet, pois não há uma rotina pré estabelecida, pois muitas vezes a roupa recolhida no centro cirúrgico acaba ficando acondicionada de forma inadequada no expurgo do laboratório de anatomia, prejudicando a 
vida útil da roupa, propiciando proliferação de microorganismos e sendo uma fonte importante de infecção e risco não só para funcionários mas para todos os usuários da Hovet.

\section{Referências}

BRASIL. Lei n. 8.080, de 19 de setembro de 1990. Lei Orgânica da Saúde. Dispõe sobre as condições para a promoção, proteção e recuperação da saúde, a organização e o funcionamento dos serviços correspondentes e dá outras providências. diário oficial da união, Brasília, 20 set. 1990.

BRASIL. Ministério do Trabalho e Emprego. Portaria n. 485, de 11 de Novembro de 2005. Dispõe sobre a Norma Regulamentadora 32 - Segurança e Saúde no Trabalho em Estabelecimentos de Assistência à Saúde. Diário Oficial da União, Brasília, 16 nov. 2005.

COSTA, E. A. vigilância sanitária: proteção e defesa da saúde. Ed. 2. São Paulo: Sobravime, 2004.

DONABEDIAN, A. Quality assessment an assurance: unity of purpose, diversity of means. inquiry. [S.1.], v. 25, n. 1, p. 173-192, 1988.

GASTAL, F. L.; ROESSLER, I. F. avaliação e qualidade. Brasília, 2006. (Módulo 2 do curso on-line: Talsa multiplicadores. Promovido pela Organização Nacional de Acreditação).

GODOY, S. C. B. et al. Riscos para o trabalhador em lavanderias hospitalares. Rev. Min. Enf. [S.l.], v. 8, n. 3, p. 382-387, jul./set. 2004.

GOLDIM, J. R. risco. Disponível em: <http://www.ufrgs.br/bioetica/risco. htm>. Acesso em: 21 maio 2012.

LUCCHESE, G. Globalização e regulação sanitária: os rumos da vigilância sanitária no Brasil. Rio de Janeiro, 2001, 329 p. Tese (Doutorado em Saúde Pública) - Escola Nacional de Saúde Pública, Fundação Osvaldo Cruz, 2001.

MURAHOVSCHI, C. H. P. et al. avaliação e qualidade. Brasília, 2006. (Módulo 3 do curso on-line Talsa multiplicadores. Promovido pela Organização Nacional de Acreditação).

PATTON, Michael Q. Qualitative evaluation methods. Beverly Hills, CA: Sage, 1980.

PROCHET,T.C. Lavanderia Hospitalar: condições e riscos para o trabalhador. nursing. [S.1.], v. 3, n. 28, p. 3234 , set. 2000.

SCHRAMM, W. Notes on case studies of instructional media projects.Working paper, the Academy for Educational Development, Washington, Dec. 1971.

YIN, Robert K. Estudo de caso: planejamento e métodos. Tradutor: Daniel Grassi. 2.ed. Porto Alegre: Bookman, 2001. 205p 